\title{
Syndrome inflammatoire multisystémique chez un adulte après une infection par le SRAS-CoV-2
}

\author{
Geneviève Kerkerian MD, Stephen D. Vaughan MD
}

Citation : CMAJ 2021 June 21;193:E956-61. doi : 10.1503/cmaj.210232-f

Voir la version anglaise de l'article ici : www.cmaj.ca/lookup/doi/10.1503/cmaj.210232

$\mathbf{U}$ $\mathrm{n}$ homme de 60 ans a consulté aux urgences pour un léger essoufflement, une grande fatigue, une anorexie et de la fièvre allant jusqu'à $40^{\circ} \mathrm{C}$ depuis 5 jours. Il a également mentionné un œdème des ganglions lymphatiques au côté gauche du cou, rentré dans l'ordre 2 jours avant qu'il ne se présente aux urgences. Quatre semaines auparavant, il avait reçu un résultat positif au test de dépistage du SRAS-CoV-2, confirmé par PCR (réaction en chaîne de la polymérase). Il n'avait aucune comorbidité connue et n'avait pas été vacciné contre le SRAS-CoV-2.

Le patient avait une fréquence cardiaque de 150 battements par minute, avec fibrillation auriculaire d'apparition récente. Sa tension artérielle était de $106 / 67 \mathrm{~mm} \mathrm{Hg}$ et sa saturation en oxygène à l'air ambiant était normale. L'examen respiratoire a montré une bonne pénétration de l'air dans les deux poumons, sans crépitements, râles ou sibilances à l'auscultation. Il présentait une conjonctivite bilatérale non purulente (figure $1 \mathrm{~A}$ ), un érythème sur la langue et une hypertrophie de celle-ci (figure 1B), un œdème à godet bilatéral et un érythème à la portion distale des orteils aux deux pieds (figure $1 \mathrm{C}$ ), sans vésicules, signes érosifs, croûtes, fissures, chaleur, enflure ni sensibilité. La radiographie pulmonaire a montré une opacification au lobe inférieur droit. Un électrocardiogramme a révélé la présence de fibrillation auriculaire avec réponse ventriculaire rapide et une anomalie diffuse non spécifique du segment ST-T (figure 2). La tomodensitométrie (TDM) du thorax avec agent de contraste n'a révélé aucune embolie pulmonaire, mais a montré une hypertrophie du côté droit du cœur et un début d'œdème pulmonaire.

Le patient a été admis, et on a commencé un traitement empirique par pipéracilline-tazobactam pour une pneumonie bactérienne possible surimposée au tableau de l'infection au SRAS-CoV-2. Les analyses de laboratoire initiales ont montré un taux élevé de protéine $\mathrm{C}$-réactive, de $\mathrm{D}$-dimères et de ferritine, ainsi qu'une neutrophilie (tableau 1), signe d'une inflammation aiguë. Son taux de troponine était élevé et il avait également un taux substantiellement augmenté de peptide B-natriurétique. Des prélèvements pour des hémocultures, une culture d'urine et un panel viral respiratoire ont été effectués et ont donné des résultats négatifs. Le patient a également obtenu des résultats

\section{Points clés}

- Le syndrome inflammatoire multisystémique est une complication rare mais grave principalement décrite chez les enfants et les adolescents après une infection par le SRASCoV-2; on peut aussi l'observer chez les personnes plus âgées.

- Après un rétablissement suite à une infection au SRAS-CoV-2, les médecins devraient soupçonner le syndrome inflammatoire multisystémique de l'adulte lorsqu'un patient présente une fièvre prolongée et une atteinte pluriorganique.

- Une élévation des taux de marqueurs inflammatoires appuie le diagnostic.

- L'instauration rapide d'un traitement immunomodulateur permet de prévenir les complications graves.

négatifs au dépistage du VIH et de l'hépatite $C$, mais était porteur d'anticorps anti-VHB. Les analyses de dépistage des maladies auto-immunes effectuées avant l'instauration du traitement étaient négatives. Le dosage sérologique des IgG pour la COVID19 s'est révélé positif. Un échocardiogramme transthoracique, effectué avant l'instauration de la corticothérapie, a montré un rythme sinusal normal et une fonction systolique et diastolique ventriculaire droite et gauche normale avec régurgitation mitrale légère à modérée. On ne disposait d'aucun échocardiogramme antérieur avec lequel comparer ces observations.

Étant donné les antécédents d'infection récente au SRAS-CoV-2 chez ce patient, ses épisodes de fièvre sans symptômes localisés, avec anomalies de la muqueuse buccale, lymphadénopathie cervicale, conjonctivite et anomalies aux membres inférieurs, nous avons soupçonné un syndrome post-COVID-19 inflammatoire. Le tableau était similaire à celui d'une complication rare mais grave signalée chez des enfants et des adolescents ayant contracté le SRAS-CoV-2, appelée syndrome inflammatoire multisystémique de l'enfant (SIME), et à celui de la maladie de Kawasaki (tableau $2^{2-4}$ ). Les équipes de rhumatologie pédiatrique et adulte responsables du patient ont convenu qu'il répondait aux critères préliminaires des CDC (Centers for Disease Control and Prevention) du syndrome inflammatoire multisystémique de l'adulte (SIMA) et de la maladie de Kawasaki, en plus de présenter plusieurs caractéristiques du SIME. 

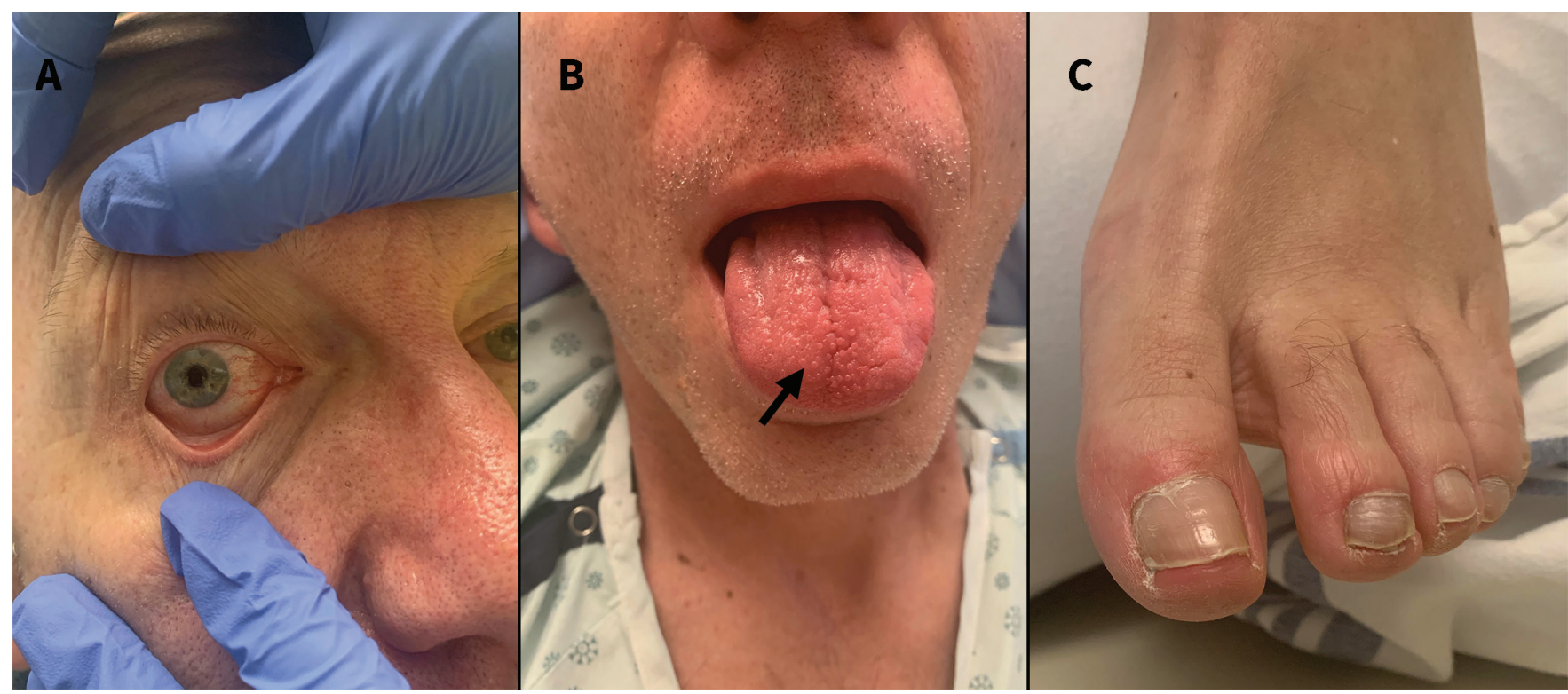

Figure 1 : A) Conjonctivite bilatérale non purulente chez un homme de 60 ans (seul l'œil droit est présenté). B) Glossite prenant la forme d'un érythème diffus non douloureux et d'un œdème symétrique de la langue. Léger énanthème du tiers antérieur du dos de la langue (petites bosses érythémateuses [flèche]) avec papilles fongiformes et filiformes hyperplasiques. C) Orteils du pied gauche montrant un érythème maculopapuleux non pâlissant. Toutes les images ont été prises avant le début du traitement.

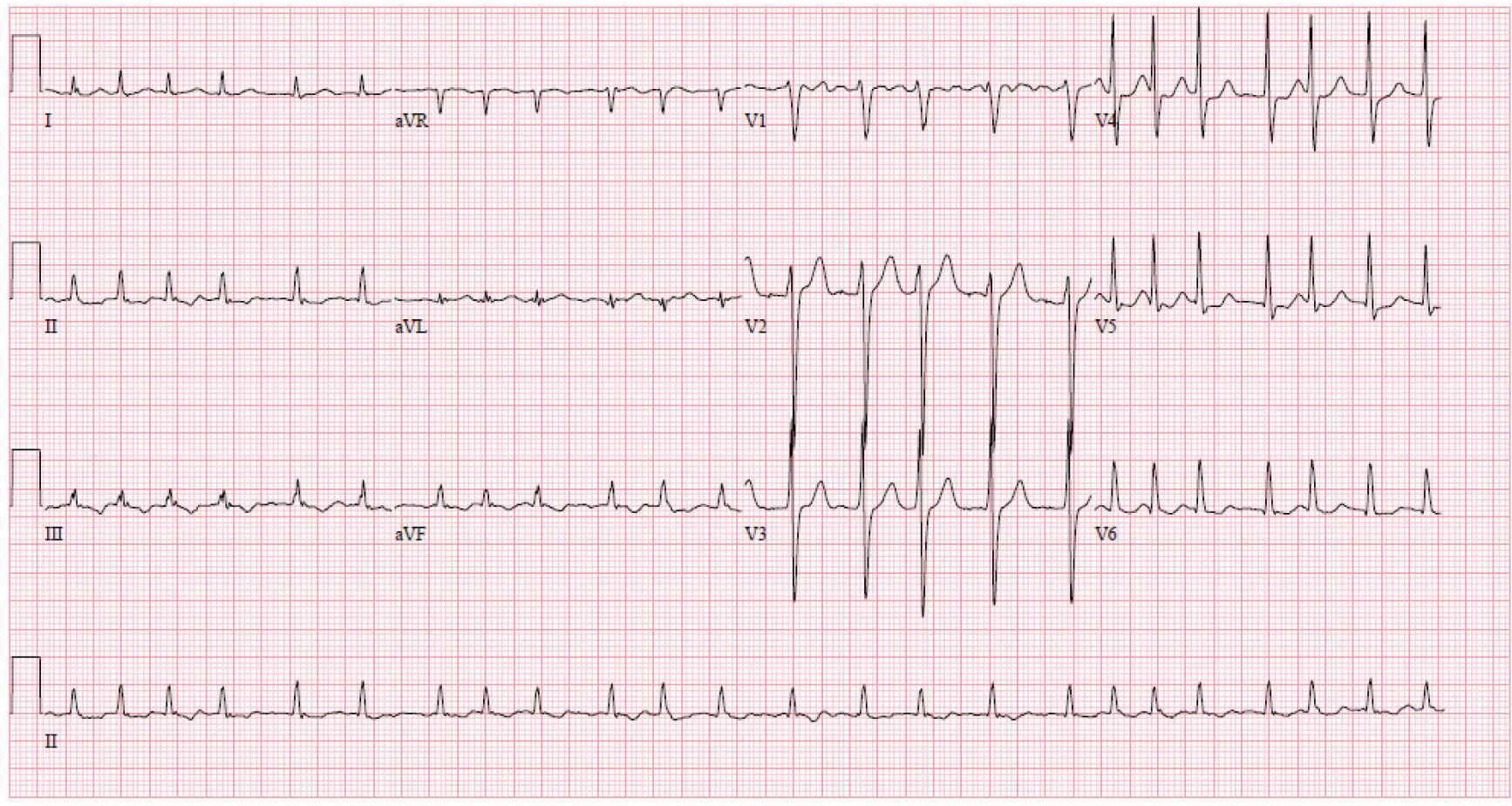

Figure 2 : Électrocardiogramme montrant une fréquence ventriculaire de 143 battements/minute, une fibrillation auriculaire avec réponse ventriculaire rapide et une anomalie diffuse non spécifique du segment ST-T.

Nous avons commencé à administrer au patient de l'acide acétylsalicylique (AAS) et de la méthylprednisolone et avons cessé les antibiotiques. Le lendemain, il a reçu des immunoglobulines intraveineuses (IgIV). En l'espace de 24 heures, il a fait état d'une amélioration substantielle de son manque d'énergie, de son essoufflement et de son anorexie, avec résolution documentée de la fièvre, amélioration de la conjonctivite (figure 3A) et diminution de l'érythème sur la langue (figure 3B), en plus d'une réduction des marqueurs inflammatoires (tableau 1). L'électrocardiogramme de contrôle s'est révélé normal (figure 4). Le patient a reçu son congé 5 jours après l'admission. Quatorze jours après son congé et à nouveau lors d'un suivi au bout de 6 semaines, son état continuait de s'améliorer sur les plans clinique et biochimique (tableau 1 ). 
Tableau 1 : Données de laboratoire chez un homme de 60 ans atteint du syndrome inflammatoire multisystémique après une infection au SRAS-CoV-2*

\begin{tabular}{|c|c|c|c|c|c|c|c|c|}
\hline Valeur de laboratoire & $\begin{array}{c}\grave{A} \\
\text { l'admission }\end{array}$ & $\begin{array}{c}\text { Jour } 1 \\
\text { post- } \\
\text { corticothérapie }\end{array}$ & $\begin{array}{l}\text { Jour } 2 \text { post- } \\
\text { corticothérapie, } \\
\text { jour } 1 \text { IgIV }\end{array}$ & $\begin{array}{l}\text { Jour } 3 \text { post- corticothérapie, } \\
\text { jour } 2 \text { IgIV, } \\
\text { jour du congé }\end{array}$ & $\begin{array}{l}6 \text { sem. } \\
\text { post- } \\
\text { congé }\end{array}$ & $\begin{array}{l}12 \text { sem. } \\
\text { post- } \\
\text { congé }\end{array}$ & $\begin{array}{l}16 \text { sem. } \\
\text { post- } \\
\text { congé }\end{array}$ & $\begin{array}{l}\text { Plage de } \\
\text { référence }\end{array}$ \\
\hline \multicolumn{9}{|l|}{ Hématologie et biochimie } \\
\hline Protéine C-réactive (mg/L) & 274,7 & & 71,6 & 35,1 & $<0,6$ & $<0,6$ & 1,5 & $0,0-8,0$ \\
\hline Ferritine $(\mu \mathrm{g} / \mathrm{L})$ & 888 & & 731 & 715 & 154 & 82 & 58 & $300-500$ \\
\hline D-dimères (mg/L FEU) & 2,14 & & 1,07 & 1,01 & 0,81 & 0,61 & 0,58 & $<0,50$ \\
\hline RIN & 1,3 & & & 1,3 & & & & $0,9-1,1$ \\
\hline Leucocytes (109/L) & 16,2 & 15,0 & 18,6 & 8,0 & 4,1 & 4,3 & 4,1 & $4,0-11,0$ \\
\hline Neutrophiles (109/L) & 14,0 & 13,3 & 16,7 & 6,2 & 1,9 & 2,0 & & $2,0-8,0$ \\
\hline Numération plaquettaire (109/L) & 248 & 375 & 505 & 464 & 202 & 218 & 179 & $150-400$ \\
\hline Lymphocytes (109/L) & 0,9 & 0,8 & 0,6 & 0,9 & 1,8 & 1,8 & & $0,7-3,5$ \\
\hline Hémoglobine (g/L) & 133 & 125 & 120 & 116 & 154 & 151 & 156 & $137-180$ \\
\hline Volume globulaire moyen (fL) & 93 & 92 & 93 & 93 & 96 & 96 & & $82-100$ \\
\hline Triglycérides (mmol/L) & & & 2,11 & & 1,65 & 1,50 & 1,67 & $0,00-1,70$ \\
\hline ALT (U/L) & 41 & & & & 14 & 9 & 8 & $8-40$ \\
\hline Créatinine $(\mu \mathrm{mol} / \mathrm{L})$ & 97 & 78 & 84 & 82 & 78 & 73 & 74 & $50-120$ \\
\hline Troponine (ng/L) & 38 & & & & & & & $0-13$ \\
\hline NT-proBNP (ng/L) & 2840 & & & & 70 & 63 & $<50$ & $0-300$ \\
\hline Lactate $(\mathrm{mmol} / \mathrm{L})$ & 2,2 & & & & & & & $0,5-2,2$ \\
\hline Créatine kinase (U/L) & 29 & & & & & & & 0-195 \\
\hline Lipase (U/L) & 17 & & & & & & & $0-80$ \\
\hline Lacticodéshydrogénase (U/L) & 199 & & & & & & & $100-235$ \\
\hline Phosphatase alcaline (U/L) & 128 & & & & & & & 30-145 \\
\hline Bilirubine totale $(\mu \mathrm{mol} / \mathrm{L})$ & 15 & & & & & & & $0-24$ \\
\hline Glucose (aléatoire) (mmol/L) & 7,1 & & & & & & & $3,3-11,0$ \\
\hline \multicolumn{9}{|l|}{ Analyse d'urine } \\
\hline Leucocytes & Trace & & & & & & & Négatif \\
\hline Nitrites & Négatif & & & & & & & Négatif \\
\hline Protéines & Négatif & & & & & & & Négatif \\
\hline Glucose & Négatif & & & & & & & Négatif \\
\hline Cétones & Négatif & & & & & & & Négatif \\
\hline Sang & Trace & & & & & & & Négatif \\
\hline Leucocytes & $6-10$ & & & & & & & $0-5 / h p f$ \\
\hline Cellules épithéliales & Modéré & & & & & & & /hpfø \\
\hline Cylindres hyalins & $5-10$ & & & & & & & $/ \mid \mathrm{pf} \|$ \\
\hline \multicolumn{9}{|l|}{ Microbiologie } \\
\hline Hémocultures & Négatif x 2 & & & & & & & Négatif \\
\hline Culture urinaire & Négatif & & & & & & & Négatif \\
\hline AAN COVID-19 $\dagger$ & $\begin{array}{l}\text { Négatif et } \\
\text { positif }{ }^{\star \star}\end{array}$ & Négatif & & & & & & Négatif \\
\hline Sérologie COVID-19‡ & Positif & & & & & & & Négatif \\
\hline Panel viral respiratoire§ & Négatif & & & & & & & Négatif \\
\hline Sérologie VIH & Négatif & & & & & & & Négatif \\
\hline Anticorps anti-VHC & Négatif & & & & & & & Négatif \\
\hline
\end{tabular}

Remarque : $\mathrm{AAN}=$ amplification des acides nucléiques, $\mathrm{ALT}=$ alanine aminotransférase, $\mathrm{FEU}=$ unité d'équivalent fibrinogène, hpf = champ à fort grossissement, IgIV = immunoglobuline intraveineuse, $\mathrm{lpf}=$ champ à faible grossissement, NT-proBNP = fraction N-terminale du peptide B-natriurétique, RIN = rapport international normalisé.

*Les valeurs en caractères gras sont anormales.

†Tous les tests d'AAN ont été faits sur des prélèvements nasopharyngés.

‡Dosage sérologique des immunoglobulines G.

§Panels viraux respiratoires pour le virus de la grippe (A et B), le virus parainfluenza (1, 2, 3, 4), les coronavirus humains (229E, NL63, OC43, HKU1), le métapneumovirus, l'entérovirus, le rhinovirus et l'adénovirus.

ฯAucune plage de référence, puisque normalement non observé.

**Le patient a subi ses tests en clinique externe le jour de son admission (résultats négatifs) puis à nouveau lorsqu'il s'est présenté aux urgences (résultats positifs). Pour le prélèvement nasopharyngé positif, la valeur de cycle seuil était de 36 (très élevée) et insuffisante pour le séquençage de la protéine S et du génome entier. Une valeur de cycle seuil plus élevée est en corrélation avec une charge virale moindre ${ }^{1}$ et le prélèvement nasopharyngé de contrôle le lendemain est revenu négatif. Cela suggère probablement que le résultat positif était un ARN résiduel de l'infection au SRAS-CoV-2 4 semaines auparavant et non pas un signe de réinfection ou de persistance de l'infection. 
Tableau 2 : Critères des CDC pour le syndrome inflammatoire multisystémique de l'adulte et les enfants ayant contracté le SRAS-CoV-2 et pour la maladie de Kawasaki ${ }^{2-4}$

\begin{tabular}{|c|c|}
\hline Caractéristiques & $\begin{array}{l}\text { Syndrome inflammatoire } \\
\text { multisystémique de l'adulte }\end{array}$ \\
\hline Définition de cas & $\begin{array}{l}\text { Hospitalisation chez un patient âgé } \\
\text { de } \geq 21 \text { ans sans signes de maladie } \\
\text { respiratoire grave ni autre } \\
\text { diagnostic plausible et atteinte d'au } \\
\text { moins un autre organe que les } \\
\text { poumons : } \\
\text { - Hypotension ou choc } \\
\text { - Dysfonction cardiaque } \\
\text { - Thromboembolie artérielle ou } \\
\text { veineuse } \\
\text { - Atteinte hépatique aiguë } \\
\text { et signes d'inflammation aiguë aux } \\
\text { analyses de laboratoire }\end{array}$ \\
\hline
\end{tabular}

Analyses et résultats de laboratoire à l'appui

\section{Syndrome inflammatoire multisystémique de l'enfant}

Maladie de Kawasaki

Patient âgé de $<21$ ans, fièvre $>38,0^{\circ} \mathrm{C}$ pendant $\geq 24 \mathrm{~h}$, ou fièvre autodéclarée durant $\geq 24 \mathrm{~h}$ et signes de maladie cliniquement graves aux analyses de laboratoire nécessitant l'hospitalisation avec atteinte pluriorganique $(\geq 2)$ :

- Cardiaque

- Rénale

- Respiratoire

- Hématologique

- Gastro-intestinale

- Dermatologique

- Neurologique

Protéine C-réactive, VSE, fibrinogène, procalcitonine, D-dimères, ferritine, lacticodéshydrogénase ou IL-6 et neutrophiles augmentés et lymphocytes et albumine diminués
Maladie de Kawasaki complète :

Fièvre $\geq 5$ jours

$E T \geq 4$ caractéristiques cliniques clés

- Anomalies affectant les membres ${ }^{\star}$

- Érythème†

- Conjonctiviteł

- Anomalies buccales§

- Lymphadénopathie cervicale (au moins $1,5 \mathrm{~cm}$ de diamètre, habituellement unilatérale)

Maladie de Kawasaki incomplète présumée : Fièvre $\geq 5$ jours

ET

2-3 critères cliniques compatibles ou nourrissons présentant de la fièvre $\geq 7$ jours sans autre explication

Protéine C-réactive $\geq 3,0 \mathrm{mg} / \mathrm{dL}$ (ou) VSE $\geq 40 \mathrm{~mm} / \mathrm{h}$

ET

1) Échocardiogramme positif : selon les critères de l'AHA ${ }^{2}$, l'échocardiogramme est considéré positif en présence de l'un ou l'autre des 3 éléments suivants :

- Score $Z$ de la descendante antérieure gauche ou de la coronaire droite $\geq 2,5$; anévrisme coronarien; ou $\geq 3$ autres caractéristiques évocatrices, y compris baisse de la fonction ventriculaire gauche, régurgitation mitrale, épanchement péricardique

- ou score Z de la descendante antérieure ou de la coronaire droite $2-2,5$

OU

2) $\geq 3$ résultats de laboratoire indicatifs (anémie pour l'âge, numération plaquettaire $\geq 450000$ après le $7^{\mathrm{e}}$ jour de fièvre, albumine $\geq 3,0 \mathrm{~g} / \mathrm{dL}$, hausse de l'alanine aminotransférase, leucocytes $\geq 15000 / \mathrm{mm}^{3}$, ou urine $\geq 10$ leucocytes/hpf)

S.O.

\section{SRAS-CoV-2 Positif pour infection au} SRAS-CoV-2 active ou antérieure (acides nucléiques, antigène ou anticorps) à l'admission ou dans les 12 sem. précédentes
Positif pour infection au SRAS-CoV-2 active ou antérieure (RT-PCR, sérologie ou test antigénique), ou exposition à un cas présumé ou confirmé de COVID-19 dans les 4 sem. précédant le déclenchement des symptômes

Remarque : AHA = American Heart Association, $\mathrm{CDC}=$ Centers for Disease Control and Prevention, $\mathrm{hpf}=$ champ à fort grossissement, IL-6 = interleukine 6, RT-PCR = test d'amplification en chaîne par polymérase couplé à une transcription inverse, S.O. = sans objet, VSE = vitesse de sédimentation des érythrocytes.

*Érythème et œdème aux mains et aux pieds durant la phase aiguë ou desquamation périunguéale durant la phase subaiguë, ou les deux.

†Érythrodermie ou érythème maculopapulaire multiforme diffus.

łInjection conjonctivale bulbaire sans exsudat.

§Érythème ou gerçure des lèvres, langue framboisée, érythème des muqueuses buccales et pharyngées.

\section{Discussion}

Depuis le début de la pandémie de SRAS-CoV-2, on a publié de nouvelles données sur la trajectoire de la maladie. Parmi les exemples de complications, mentionnons la thromboembolie veineuse, la maladie cardiovasculaire, l'atteinte rénale ou hépatique aiguë, les symptômes neurologiques et le syndrome post-
COVID-195. Le syndrome inflammatoire multisystémique de l'enfant a d'abord été décrit en avril 2020 sous la forme d'un syndrome hyperinflammatoire présentant des caractéristiques semblables à celles de la maladie de Kawasaki ${ }^{2,3,6}$. En date du 3 mai 2021, 3742 enfants aux États-Unis avaient officiellement reçu un diagnostic de SIME, selon les critères résumés au tableau 2, et 35 décès y avaient été associés ${ }^{7}$. 


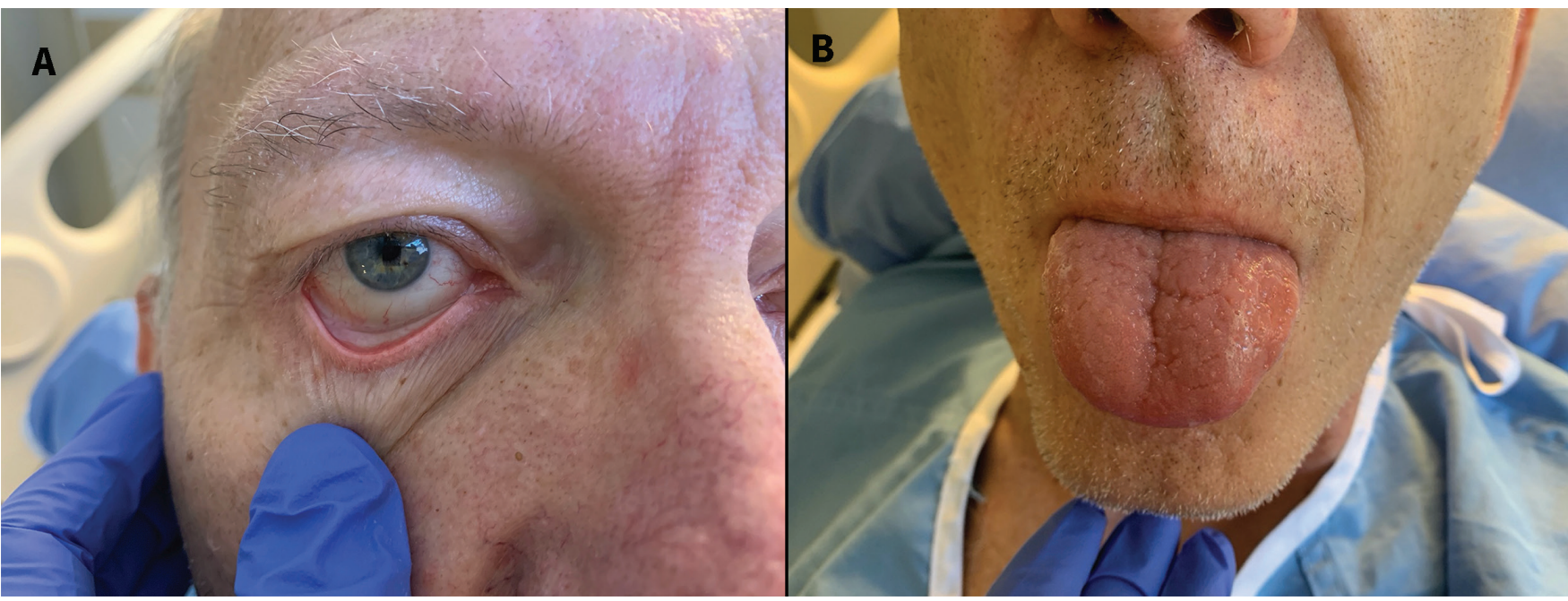

Figure 3 : Résolution quasi-complète de la conjonctivite du patient (A), amélioration de la glossite et quasi-résolution de l'énanthème de la langue (B) au jour 1 après l'instauration de la corticothérapie à dose élevée.

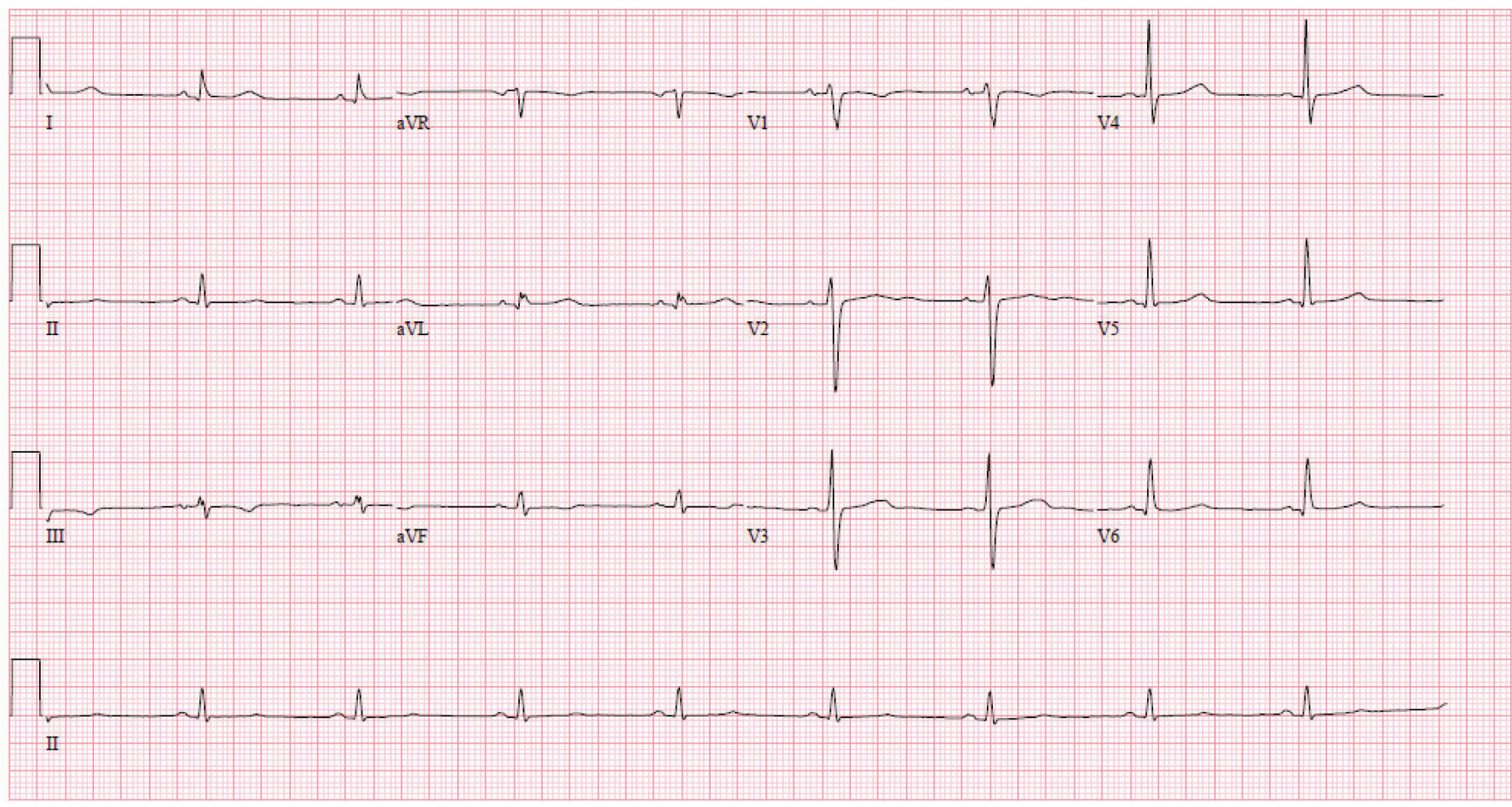

Figure 4 : Bradycardie sinusale à 54 battements/minute et électrocardiogramme par ailleurs normal, avec résolution de la fibrillation auriculaire et des anomalies du segment ST-T avant le congé.

En octobre 2020, les CDC ont publié une revue de 27 cas correspondant à la description du syndrome inflammatoire multisystémique chez des adultes ${ }^{4}$. La définition de cas préliminaire du SIMA est présentée au tableau 2, et les cas décrits à ce jour affectent des patients de moins de 50 ans. À mesure que nous continuons de découvrir le SIMA, il est toutefois prudent de ne pas présumer d'un seuil d'âge pour établir un diagnostic, comme l'illustre le présent cas $^{4,8-10}$.

De nombreuses questions au sujet du SIMA restent sans réponses. Notre patient n'en a pas présenté les symptômes les plus fréquents, soit l'AVC, le choc ou la dysfonction cardiaque nécessitant une prise en charge dans une unité de soins intensifs ${ }^{4}$. Cela pourrait s'expliquer par le fait que le spectre de sa maladie était plus bénin ou par la rapidité du diagnostic et des interventions. Plusieurs caractéristiques de ce cas ont rapidement éveillé les soupçons quant au SIMA ou à la maladie de Kawasaki. Notre patient répondait aux critères diagnostiques de la maladie de Kawasaki complète ( 5 jours de fièvre et 4 des 5 caractéristiques cliniques suivantes: anomalies des membres, conjonctivite, anomalies buccales et lymphadénopathie cervicale; il n'a pas présenté d'éruption cutanée) et du SIME (sauf l'âge). Toutefois, les données récentes sur l'infection au SRAS-CoV-2 et l'âge du patient ont fait du SIMA l'étiologie la plus probable chez ce patient. 
L'American College of Rheumatology a publié une revue comparant et mettant en parallèle les caractéristiques du SIME et de la maladie de Kawasaki, et a relevé que $25 \%-50 \%$ des patients qui répondaient aux critères du SIME répondaient aussi à tous les critères diagnostiques de la maladie de Kawasaki ${ }^{11}$. Deux différences entre ces maladies sont la distribution ethnique (incidence de la maladie de Kawasaki plus élevée chez les personnes d'origine japonaise) et l'âge (habituellement moins de 5 ans pour la maladie de Kawasaki, et un éventail plus vaste allant de 3 mois à 21 ans pour le SIME) 2,11,12.

On n'a pas encore établi de traitement standard pour le SIMA. L'état de notre patient s'est amélioré en 3 jours avec l'administration de méthylprednisolone à $125 \mathrm{mg} / \mathrm{j}$, puis de prednisone orale à dose moindre, soit $50 \mathrm{mg} / \mathrm{j}$, pendant 3 jours, après quoi on a diminué sa dose de $10 \mathrm{mg} / \mathrm{j}$ tous les 3 jours jusqu'au sevrage de la corticothérapie (pour un cycle oral total de 15 jours). Il a reçu $1 \mathrm{~g} / \mathrm{kg} / \mathrm{j}$ d'IgIV pendant 2 jours et $325 \mathrm{mg} / \mathrm{j}$ d'AAS par voie orale jusqu'à résolution des symptômes cliniques, soit l'absence de fièvre et le retour à la normale du taux de protéine C-réactive. Ce traitement se basait sur l'opinion d'experts et sur les données les plus récentes au sujet de la maladie de Kawasaki et du SIME',6,8,11-13. L'American College of Rheumatology recommande une approche séquentielle pour le traitement immunomodulateur du SIME, avec IgIV ou corticothérapie à envisager en première intention ${ }^{11}$, et l'American Heart Association recommande une dose élevée simple d'IgIV (2 g/kg) pour le traitement de la maladie de Kawasaki². Même si le patient n'a pas présenté de thrombocytose cliniquement significative, nous avons prescrit un cycle bref d'AAS à dose élevée pour prévenir la thrombose et les anévrismes coronariens ${ }^{11}$.

Le suivi optimal du SIMA n'a pas encore été établi, mais les experts appuient la surveillance des anévrismes et de la dilatation des coronaires, comme pour le SIME ou la maladie de Kawasaki²,11. Chez les enfants, l'échocardiogramme est la modalité d'imagerie à privilégier pour mesurer le diamètre des coronaires, mais la visualisation des coronaires devient progressivement plus difficile à mesure que le corps croît. On préfère donc la coronarographie par TDM pour la surveillance chez les adultes ${ }^{2}$. Chez notre patient, la coronarographie a montré une atteinte coronaire tritronculaire, sans sténose cliniquement importante ni anévrismes coronariens ou signes de vascularite des coronaires. Au moment de rédiger ce rapport, nous attendions les résultats de la TDM de contrôle pour visualiser le calibre des coronaires 6 mois après le congé.

Contrairement au SIME, le SIMA ne fait pas partie actuellement des diagnostics qu'il faut obligatoirement déclarer aux autorités sanitaires des provinces ou des États, mais cela devrait être encouragé pour faciliter la recherche et améliorer l'issue de la maladie chez les patients ${ }^{6}$.

\section{Références}

1. Ontario Agency for Health Protection and Promotion (Public Health Ontario). An overview of cycle threshold values and their role in SARS-CoV-2 real-time PCR test interpretation. Toronto: Queen's Printer for Ontario; 2020. Accessible ici : www.publichealthontario.ca/-/media/documents/ncov/main/2020/09/ cycle-threshold-values-sars-cov2-pcr.pdf?la=en (consulté le 11 mai 2021).

2. McCrindle BW, Rowley AH, Newburger JW, et al.; American Heart Association Rheumatic Fever, Endocarditis, and Kawasaki Disease Committee of the Council on Cardiovascular Disease in the Young; Council on Cardiovascular and Stroke Nursing; Council on Cardiovascular Surgery and Anesthesia; Council on Epidemiology and Prevention. Diagnosis, treatment, and long-term management of Kawasaki disease: a scientific statement for health professionals from the American Heart Association. Circulation 2017;135:e927-99.

3. National Center for Immunization and Respiratory Diseases (NCIRD). Information for healthcare providers about multisystem inflammatory syndrome in children (MIS-C). Atlanta: Centers for Disease Control and Prevention; reviewed 2021 Feb. 17. Accessible ici : https://www.cdc.gov/mis-c/hcp/\#: :text=Case\%20Definition \%20for\%20MIS\%2DC\&text=No\%20alternative\%20plausible\%20diagnoses\%3B\% 20AND,to\%20the\%20onset\%20of\%20symptoms (consulté le 13 janv. 2021).

4. Morris SB, Schwartz NG, Patel P, et al. Case series of multisystem inflammatory syndrome in adults associated with SARS-CoV-2 Infection - United Kingdom and United States, March-August 2020. MMWR Morb Mortal Wkly Rep 2020;69: 1450-6.

5. Complications. In: BMJ Best Practice: Coronavirus diseases 2019 (COVID-19). London (UK): BMJ; 2021. Accessible ici : bestpractice.bmj.com/topics/en-us /3000168/pdf/3000168/Coronavirus\%20disease\%202019\%20\%28COVID-19\%29 .pdf (consulté le 11 janv. 2021).

6. Cheung EW, Zachariah P, Gorelik M, et al. Multisystem inflammatory syndrome related to COVID-19 in previously healthy children and adolescents in New York City. JAMA 2020;324:294-6.

7. National Center for Immunization and Respiratory Diseases (NCIRD). Health department-reported cases of multisystem inflammatory syndrome in children (MIS-C) in the United States. Atlanta: Centers for Disease Control and Prevention; reviewed 2021 May 11. Accessible ici : https://www.cdc.gov/mis-c/cases/index. html (consulté le 10 janv. 2021).

8. Kofman AD, Sizemore EK, Detelich JF, et al. A young adult with COVID-19 and multisystem inflammatory syndrome in children (MIS-C)-like illness: a case report. BMC Infect Dis 2020;20:716.

9. Shaigany S, Gnirke M, Guttmann A, et al. An adult with Kawasaki-like multisystem inflammatory syndrome associated with COVID-19. Lancet 2020;396:e8-10.

10. Ahsan T, Rani B. A case of multisystem inflammatory syndrome post-COVID-19 infection in an adult. Cureus 2020;12:e11961.

11. Henderson LA, Canna SW, Friedman KG, et al. American College of Rheumatology clinical guidance for multisystem inflammatory syndrome in children associated with SARS-CoV-2 and hyperinflammation in pediatric COVID-19: version 1. Arthritis Rheumatol 2020;72:1791-805.

12. Gomard-Mennesson E, Landron C, Dauphin C, et al. Kawasaki disease in adults: report of 10 cases. Medicine (Baltimore) 2010;89:149-58.

13. Tam H, El Tal T, Go E, et al. Pediatric inflammatory multisystem syndrome temporally associated with COVID-19: a spectrum of diseases with many names. CMAJ 2020;192:E1093-6.

Intérêts concurrents : Aucun déclaré.

Cet article a été révisé par des pairs.

Les auteurs ont obtenu le consentement du patient.

Affiliation : Division infectiologie, Département de médecine, Faculté de médecine Cumming, Université de Calgary, Calgary, Alb.

Collaborateurs : Les deux auteurs ont contribué à la conception du travail, ont rédigé le manuscrit et en ont révisé de façon critique le contenu intellectuel important; ils ont donné leur approbation finale pour la version destinée à être publiée et assument l'entière responsabilité de tous les aspects du travail.

Propriété intellectuelle du contenu : Il s'agit d'un article en libre accès distribué conformément aux modalités de la licence Creative Commons Attribution (CC BY-NC-ND 4.0), qui permet l'utilisation, la diffusion et la reproduction dans tout médium à la condition que la publication originale soit adéquatement citée, que l'utilisation se fasse à des fins non commerciales (c.-à-d., recherche ou éducation) et qu'aucune modification ni adaptation n'y soit apportée. Voir : https://creativecommons.org/licenses/by-nc-nd/4.0/deed.fr.

Remerciements : Les auteurs remercient les $D^{\text {rs }}$ Glen Hazlewood, Heinrike Schmeling et Kevin Fonseca pour leur opinion et leur expertise lors de la prise en charge de ce cas.

Correspondance : Geneviève Kerkerian, genevieve.kerkerian@albertahealthservices.ca 